\title{
Morphological and Pathogenic Variability among Rhizoctonia bataticola Isolates Associated with Soybean (Glycine max L.) from India
}

\author{
R.M. Gade*, Y.K. Belkar and Y.V. Ingle \\ Department of Plant Pathology, Dr. Panjabrao Deshmukh Krishi Vidyapeeth, \\ Akola (M.S) 444 104, India \\ *Corresponding author
}

A B S T R A C T

\begin{tabular}{|l|}
\hline Ke y w o r d s \\
Rhizoctonia root \\
rot, Soybean, \\
Pathogenic \\
variability
\end{tabular}

Rhizoctonia bataticola is a serious pathogen of many crops. In the present studies, 40 isolates of Rhizoctonia bataticola from different agro climatic regions of India were analyzed for morphological and pathogenic variability. Regardless of their geographic origins, significant differences were detected among 40 isolates in their radial growth, sclerotial size, and shape as well as in pathogenicity. Thirty two isolates were rated as fast growing and the rest of the isolates as medium growing. Sixteen isolates were classified as large sized, 8 as small sized, and the remaining 16 isolates as medium sized on the basis of their sclerotial size. On the basis of disease expression, isolate Rb-29 (Kurundwad, Sangli) showed broad virulence range as it infected all the genotypes followed by isolate $\mathrm{Rb}-38$ (Udaipur, Rajasthan) which infected eleven genotypes. No relationship was found among the morphological characters and pathogenicity of the isolates. These morphological and pathogenic variations in various isolates of $R$. bataticola may be considered important in disease management systems and will be useful in breeding programmes of soybean cultivars resistant to root rot.

\section{Introduction}

Soybean [Glycine max (L.) Merill] is the major oilseed crop in the world and is cultivated on an area of 108.83 Lakh ha with a total production of 114.90 Lakh MT. The crop is grown in a wide range of agro climatic zones. The low yield of soybean in India can be attributed to legions of biotic and abiotic constraints. Among biotic factors, diseases are the most dominant. Soybean crop can be attacked by more than 100 pathogens (Sinclair and Shurlleff, 1975). In India, losses due to various diseases are estimated as 12 per cent of total production. The diseases include rust, wilts, leaf spot, rots and powdery mildew, bacterial and viral diseases. Among these, root rot caused by Rhizoctonia bataticola is of prime importance in reducing crop yield.

Yield losses 30-50 per cent due to Macrophomina phaseolina in soybean crop has been reported (Yang and Navi, 2005). Rhizoctonia bataticola reduces the yield of soybean by 2-21 per cent (Wrather and Koenning, 2006). The pathogen is distributed in diverse climatic conditions from arid to tropical regions and has a broad host range. 
There are more than 500 hosts of the fungus including legume and cereal plants (Dhingra and Chagas, 1981; Sinclair, 1982). $M$. phaseolina is a soil- and seed-borne pathogenic fungus and produces cushion shaped black sclerotia (Wheeler, 1975). Its prevalence can be enhanced by different physiological and ecological factors such as low moisture contents, high temperature, and heat (Papavizas, 1977; Dhingra and Sinclair, 1978). Disease severity is correlated with viable sclerotia present in the soil. Charcoal rot infects plants at almost all growth stages. Dark lesions appear on the epicotyls and hypocotyls followed by seedling death due to obstruction of xylem vessels. In plants, the pathogen causes red to brown lesions on roots and stems with production of dark mycelia and black microsclerotia. Ultimately the plant becomes defoliated and wilted (Abawi and Pastor-Corrales, 1990) and perishes.

Among the main management strategies, use of cultivars resistant to $M$. phaseolina has gained wide popularity and acceptance amongst farmers as application of fungicides is often intertwined with potential hazards to humans and the environment. Furthermore, resistant cultivars outstrip fungicides in various respects and emphasis is being laid on the development of new resistant germplasm. However, it has been observed that control measures against pathogens become complicated and even ineffective due to the variability among populations of the same pathogen in different agro ecological zones.

There are reports in other parts of the world that populations of $M$. phaseolina showed significant variations morphologically, physiologically, pathogenically and genetically. These variations aid the pathogen to adapt and survive in diverse environments. A thorough knowledge of pathogenic variability of $R$. bataticola is essential to design disease management strategies for different agro climatic zones of the country by breeding resistant cultivars. Hence, we investigated morphological and pathogenic variability among 40 isolates of $R$. bataticola infecting soybean, collected from five different major soybean growing states of India. It has also been determined whether morphological variations among $M$. phaseolina isolates have any relationship with the pathogenic variability.

\section{Materials and Methods}

\section{Collection of fungal isolates}

A total of 40 isolates of Rhizoctonia bataticola were collected from 5 major soybean growing states viz. Madhya Pradesh, Maharashtra, Karnataka, Rajasthan and Andhra Pradesh of the India representing different agro climatic zones of country delineated mainly on the basis of physiographic and climatic characteristics, soil type, and agricultural land use (Table 1). Samples of stems bearing microsclerotia of the fungus and characteristic symptoms of root rot were collected from the infected soybean plants from farmers' fields and designated. The diseased samples were first packed in paper bags and then in $15 \times 20$ $\mathrm{cm}$ polyethylene bags, labeled, brought to the lab, and stored at $4{ }^{\circ} \mathrm{C}$ until processed for identification.

\section{Isolation, purification, and identification of R. bataticola}

The fungus was isolated from stem bark tissues of soybean bearing fungal sclerotia and showing characteristic root rot symptoms. The samples were cut into small pieces $(5-10 \mathrm{~mm}$ long) and surface sterilized with 1:1000 mercuric chloride for 30 seconds and then rinsed thrice in sterilized distilled water. The pieces were placed on potato dextrose agar medium in Petri dishes and incubated in dark at $27 \pm 2^{\circ} \mathrm{C}$ for 7 days. A small portion of the 
fastest growing colony of $R$. bataticola was taken from the periphery of a $90 \mathrm{~mm}$ diameter Petri dish, spread onto Petri dishes containing potato dextrose agar medium and incubated in the dark at $27 \pm 2{ }^{\circ} \mathrm{C}$ for 7 days. A small portion of the colony having sclerotia was taken up into a drop of sterilized water and agitated with a sterilized needle to separate the sclerotia from the mycelia. Sclerotia were then transferred to $90 \mathrm{~mm}$ diameter Petri dishes containing potato dextrose agar medium. Colonies appearing from single sclerotium were again transferred to potato dextrose agar medium in $90 \mathrm{~mm}$ Petri plates, incubated as mentioned above and identified as described.

\section{Storage of pure cultures of $\boldsymbol{R}$. bataticola}

The purified culture (5 mm disc) from each isolate growing on PDA was transferred to10 $\mathrm{ml}$ culture tubes and incubated in the dark at $27 \pm 2{ }^{\circ} \mathrm{C}$ for 6 days, until the surface of PDA was covered with a dense sclerotial layer of the fungal culture. The culture tubes were labeled and stored at $4^{\circ} \mathrm{C}$.

\section{Multiplication of $R$. bataticola}

Ground sorghum seeds were water-soaked overnight, air-dried under room temperature, and placed in conical flasks. The mouth of each flask was plugged with cotton wool, wrapped in aluminum foil, and autoclaved at 15 psi $\left(121^{\circ} \mathrm{C}\right)$ for 20 minutes. After cooling, the seeds in flasks were inoculated with $4 \mathrm{~mm}$ mycelial plugs from a 7-day old culture of $R$. bataticola and incubated at $27 \pm 2^{\circ} \mathrm{C}$ for 15 days. The flasks were shaken at alternate days for uniform colonization of the grains. The inoculum thus produced was used in pot assay.

\section{Determination of morphological variability}

Morphological variability among 40 isolates of $R$. bataticola was studied on the basis of the following parameters.

\section{Radial growth}

For studying variability in radial growth, the isolates were grown on Potato Dextrose Agar. Fifteen milliliters of autoclaved PDA was poured in $90 \mathrm{~mm}$ diameter Petri plates, allowed to solidify, and inoculated in the center with a $5 \mathrm{~mm}$ plug from the actively growing culture of each isolate of the fungus. The plates were incubated at $27 \pm 2^{\circ} \mathrm{C}$ for 7 days. Each isolate was replicated three times. After the stipulated period the growth of each isolate was measured in terms of colony diameter and their means were computed. On the basis of radial growth, the isolates were categorized as fast $(>80 \mathrm{~mm})$, and medium (70-80 $\mathrm{mm})$ growing.

\section{Sclerotial size}

For measuring sclerotial size, slides from 7day-old pure cultures of $R$. bataticola isolates were prepared and examined under a microscope ocular micrometer. Sizes of fifty randomly selected sclerotia were measured and their means were calculated. The isolates were classified as large $(>80 \mu \mathrm{m})$, medium $(60-80 \mu \mathrm{m})$, and small $(<60 \mu \mathrm{m})$ sized.

\section{Determination of pathogenic variability}

The pathogenicity of 40 isolates was studied on susceptible soybean cultivar TAMS-38 in the greenhouse with six replications for each isolate. Seeds were disinfected by immersing in 0.1 per cent mercuric chloride for $1 \mathrm{~min}$, rinsed in sterilized water, and air-dried. Ten seeds of cultivar TAMS-38 of soybean were sown in pots containing $2 \mathrm{~kg}$ soil infested with each isolate of $R$. bataticola in 1:9 proportions (inoculum + soil). Pots without inoculum served as controls. The pots were placed in a greenhouse at $30 \pm 2^{\circ} \mathrm{C}$. The pots were watered as and when required and observations on occurrence of root rot were recorded. On the basis of occurrence and 
symptoms, the isolates were identified as pathogenic.

\section{Grouping of isolates}

Forty isolates of $R$. bataticola were tested by sick soil method for their virulence against susceptible variety TAMS-38. The percent root rot was recorded on the basis of healthy and root rot infected plants. The isolates of $\mathrm{R}$. bataticola were tentatively divided into five groups on the basis of virulence as follows (Pawar, 2010).

Category Per cent mortality due to root rot

Non-pathogenic

$0 \%$

Weakly pathogenic

$1-20 \%$

Moderately pathogenic

$21-50 \%$

Strongly pathogenic

$51-70 \%$

Highly pathogenic

$>70 \%$

\section{Host differential reaction (Pot culture)}

Virulence analysis of $R$. bataticola isolates was carried out on set of twelve host differentials showing absolutely resistant, moderately resistant, moderately susceptible, susceptible and highly susceptible reactions viz., GBIC-18758, AMS-595, AMS-MB-5-18, AMS-MB-5-19, AMS-1003, AMS-475, AMS3923, AMS-9933, Bragg, Punjab-1, TAMS-38 and TAMS-9821 in green house. Seven isolates of $R$. bataticola collected from different locations showing different pathogenic reactions were selected and inoculated separately to each host differential. Ten seeds of each cultivar were sown in triplicate in surface sterilized pots filled with 2 $\mathrm{kg}$ sterilized soil, inoculated with 14 days old culture @ $100 \mathrm{~g} \mathrm{~kg}^{-1}$ of the pathogen multiplied on sand sorghum grain medium seven days before sowing. The pots without inoculum were served as control. The incidence of root rot was recorded at 15 days interval up to crop maturity. Reactions were graded according to the uniform method of disease rating as absolutely resistant (No mortality), highly resistant (0.01-11.11\% mortality), moderately resistant (12.22-33.33 $\%$ mortality), moderately susceptible (34.44$55.55 \%$ mortality), susceptible (56.77-77.77 $\%$ mortality) and highly susceptible (78.88$100.00 \%$ mortality).

\section{Results and Discussion}

\section{Morphological variability among Rhizoctonia bataticola isolates}

Significant variations were observed in the morphological parameters among 40 isolates of $R$. bataticola collected from different major soybean producing states of India.

\section{Radial growth}

Significant differences among 40 isolates of $R$. bataticola collected from different states were observed on the basis of radial growth. The individual average radial growths of 40 isolates of $R$. bataticola ranged from 75.00 to $90.00 \mathrm{~mm}$. Maximum colony diameters of 90.00 and $85.66 \mathrm{~mm}$ were observed in case of isolate Rb-1, Rb-6, Rb-11, Rb-12, Rb-15, Rb$22 \mathrm{Rb}-23, \mathrm{Rb}-29, \mathrm{Rb}-37$ and $\mathrm{Rb}-38$, and proving to be the fast growing, while isolates $\mathrm{Rb}-5$ and $\mathrm{Rb}-26$ showed the minimum radial growths and were rated as medium growing. The individual radial growths of all the isolates are shown in Table 2. Thirty two isolates showed radial growths above $80 \mathrm{~mm}$ and were rated as fast growing while rest of the isolates showed growth between 70 and 80 $\mathrm{mm}$ and hence were classified as medium growing (Table 3).

\section{Sclerotial size}

Significant variations were also observed among these isolates regarding the size of their sclerotia. Maximum sclerotial size was 
observed in case of isolate Rb-33 (120.11 $\mu \mathrm{m})$ while the isolate $\mathrm{Rb}-40$ produced the smallest size sclerotia $(42.03 \mu \mathrm{m})$. The individual average sclerotial sizes of isolates ranged from 42.03-120.11 $\mu \mathrm{m}$ which are given in Table 2.

The size of sclerotia of 16 isolates was above $80 \mu \mathrm{m}$ and was classified as large sized while 16 isolates with sclerotial size less than $60 \mu \mathrm{m}$ were rated as small sized. The remaining 8 isolates ranged between 60 and $80 \mu \mathrm{m}$ sclerotial size and were categorized as medium sized (Table 4).

\section{Pathogenic variability among $\boldsymbol{R}$. bataticola} isolates

Pathogenic ability of isolates indicated that out of 40 isolates of $R$. bataticola, all isolates proved pathogenic with susceptible cultivar TAMS-38 (Table 5). Among different isolates tested, two viz., Rb-3 and Rb-17 were highly pathogenic with 71-100 per cent root rot incidence whereas eight viz., Rb-10, Rb-13, Rb-18, Rb-22, Rb-29, Rb-34, Rb-39 and Rb40 were strongly pathogenic with average of 51-70 per cent root rot. Isolates, $\mathrm{Rb}-4, \mathrm{Rb}-5$, Rb-8, Rb-9, Rb-11, Rb-12, Rb-14, Rb-15, Rb16, Rb-20, Rb-21, Rb-23, Rb-24, Rb-25, Rb26, Rb-27, Rb-28, Rb-30, Rb-31, Rb-33, Rb$35, \mathrm{Rb}-36, \mathrm{Rb}-37$ and $\mathrm{Rb}-38$ were moderately pathogenic showing 21 to 50 per cent root rot and remaining six viz.,Rb-1, Rb-2, Rb-6, Rb7, Rb-19 and Rb-32 were weakly pathogenic with average root rot incidence of 1 to 20 per cent. However, none of the isolate proved to be non-pathogenic to cultivar TAMS-38.

\section{Grouping of isolates}

Forty isolates of R. bataticola were tested by sick soil method for their virulence against susceptible cultivar TAMS-38. The per cent root rot was recorded on the basis of healthy and root rot infected plants. The data on pathogenic variability in different isolates of
R. bataticola are given in Table 5. The isolates were tentatively divided in five groups based on their pathogen ability (Table 6).

\section{Host differential reaction (Pot culture)}

The pathogenic reactions of seven isolates of $R$. bataticola against a set of twelve genotypes of soybean viz., GBIC-18758, AMS-595, AMS-MB-5-18, AMS-MB-5-19, AMS-1003, AMS-475, AMS-3923, AMS-9933, Bragg, Punjab-1, TAMS-38 and TAMS-98-21 were assessed under sick soil conditions.

Significant differences among isolates, varieties, and their interactions in which the disease response on various cultivars varied between 3-9 grades shown in Table 7.

Genotype GBIC-18758 was found highly resistant against isolate $\mathrm{Rb}-17(9.52 \%)$ and moderately susceptible against Rb-3 (46.15\%) and Rb-10 (35.29\%).

Genotype AMS-595 was moderately susceptible to Rb-17 (46.15\%) and highly susceptible to isolates Rb-29 (100\%), Rb-3 $(83.33 \%)$ and $\mathrm{Rb}-10$ (80 \%) while isolates Rb-39 (75.00 \%), Rb-38 (76.47 \%) and Rb-22 $(66.67 \%)$ exhibited susceptible reaction.

AMS-MB-5-18 showed moderate resistance against Rb-17 (21.74 \%) and Rb-22 (31.25\%) whereas moderately susceptible reaction against Rb-38 (53.85 \%) and Rb-29 (50\%). Isolates, Rb-38 (50 \%), Rb-10 (50\%), Rb-29 (46.15\%), Rb-3 (47.37\%) and Rb-17 (45.00 $\%)$ exhibited moderately susceptible reaction towards genotype AMS-MB-5-19 while moderately resistant reaction against $\mathrm{Rb}-39$ $(16.67 \%)$.

Isolates $\mathrm{Rb}-38,(18.18 \%)$ and $\mathrm{Rb}-10$ (21.43 $\%$ ) were least virulent as moderately resistant reaction was developed against genotype AMS-1003. 
Table.1 Isolates of Rhizoctonia bataticola collected from soybean plants from different agro climatic regions

\begin{tabular}{|c|c|c|c|c|c|c|}
\hline Sr No & State & Agro Climatic Regions & Soil Type & $\begin{array}{l}\text { Rainfall } \\
\text { (mm) }\end{array}$ & Districts & Isolates \\
\hline \multirow[t]{6}{*}{1} & \multirow{6}{*}{$\begin{array}{l}\text { Madhya } \\
\text { Pradesh }\end{array}$} & \multirow[t]{3}{*}{ Malwa Plateau } & \multirow{3}{*}{$\begin{array}{l}\text { Medium Black } \\
\text { (Medium) }\end{array}$} & \multirow[t]{3}{*}{$800-1200$} & Indore & $\mathrm{Rb}-1, \mathrm{Rb}-2, \mathrm{Rb}-3$ \\
\hline & & & & & Dewas & $\begin{array}{c}\mathrm{Rb}-4, \mathrm{Rb}-5, \mathrm{Rb}-6, \mathrm{Rb}-7 \\
\mathrm{Rb}-8\end{array}$ \\
\hline & & & & & Ujjain & Rb-9, Rb-10, Rb-11 \\
\hline & & Nimar Plains & $\begin{array}{l}\text { Medium Black } \\
\text { (Medium) }\end{array}$ & 800-1000 & Harda & Rb-12, Rb-13, Rb-14 \\
\hline & & Vindhya Plateau & $\begin{array}{c}\text { Medium Black and } \\
\text { Deep Black }\end{array}$ & $1200-1400$ & Sehore & $\mathrm{Rb}-15, \mathrm{Rb}-16, \mathrm{Rb}-17$ \\
\hline & & $\begin{array}{l}\text { Kymore Plateau and } \\
\text { Satpura Hills }\end{array}$ & $\begin{array}{l}\text { Mixed Red and Black } \\
\text { soils (Medium) }\end{array}$ & $1000-1400$ & Jabalpur & $\mathrm{Rb}-18, \mathrm{Rb}-19, \mathrm{Rb}-20$ \\
\hline \multirow[t]{8}{*}{2} & \multirow[t]{8}{*}{ Maharashtra } & \multirow{3}{*}{$\begin{array}{c}\text { Central Maharashtra } \\
\text { Plateau }\end{array}$} & \multirow[t]{3}{*}{ Black to Red } & \multirow[t]{3}{*}{$700-900$} & Akola & $\mathrm{Rb}-21$ \\
\hline & & & & & Amaravati & $\mathrm{Rb}-22$ \\
\hline & & & & & Buldhana & $\mathrm{Rb}-24$ \\
\hline & & \multirow{2}{*}{$\begin{array}{c}\text { Central Vidharbha } \\
\text { Region }\end{array}$} & \multirow{2}{*}{$\begin{array}{l}\text { Black soils, medium to } \\
\text { heavy texture }\end{array}$} & \multirow{2}{*}{1130} & Wardha & $\mathrm{Rb}-23$ \\
\hline & & & & & Nagpur & $\mathrm{Rb}-25$ \\
\hline & & \multirow{3}{*}{$\begin{array}{l}\text { Western Maharashtra } \\
\text { Plain Zone }\end{array}$} & \multirow[t]{3}{*}{ Greyish Black } & \multirow[t]{3}{*}{$700-1200$} & Satara & $\mathrm{Rb}-26$ and $\mathrm{Rb}-27$ \\
\hline & & & & & Sangli & $\mathrm{Rb}-28$ and $\mathrm{Rb}-29$ \\
\hline & & & & & Kolhapur & $\mathrm{Rb}-30$ and $\mathrm{Rb}-31$ \\
\hline \multirow[t]{2}{*}{3} & \multirow[t]{2}{*}{ Karnataka } & \multirow[t]{2}{*}{ Northern Dry Zone } & \multirow[t]{2}{*}{ Black Clay Medium } & \multirow[t]{2}{*}{ 464-785 } & Belgaun & $\mathrm{Rb}-32, \mathrm{Rb}-33, \mathrm{Rb}-34$ \\
\hline & & & & & Dharwad & $\mathrm{Rb}-35$ and $\mathrm{Rb}-36$ \\
\hline \multirow[t]{3}{*}{4} & \multirow[t]{3}{*}{ Rajasthan } & \multirow{2}{*}{$\begin{array}{l}\text { Sub Humid Southern } \\
\text { Plains }\end{array}$} & \multirow[t]{2}{*}{ Alluvial } & \multirow[t]{2}{*}{$500-900$} & Bhilwara & $\mathrm{Rb}-38$ \\
\hline & & & & & Udaipur & $\mathrm{Rb}-39$ \\
\hline & & $\begin{array}{c}\text { Humid South Eastern } \\
\text { Plains }\end{array}$ & Black of Alluvial origin & $650-1000$ & Kota & $\mathrm{Rb}-37$ \\
\hline 5 & Telangana & North Telangana Zone & Red Sandy soils & $900-1500$ & Adilabad & $\mathrm{Rb}-40$ \\
\hline
\end{tabular}


Int.J.Curr.Microbiol.App.Sci (2018) 7(1): 2575-2588

Table.2 Morphological variations among different isolates of Rhizoctonia bataticola

\begin{tabular}{|c|c|c|c|c|}
\hline Sr. No & Isolates & $\begin{array}{l}\text { Radial growth } \\
\text { (mm)* }\end{array}$ & $\begin{array}{l}\text { Mean sclerotial size } \\
(\mu \mathrm{m})^{* * *}\end{array}$ & Shape of sclerotia \\
\hline 1 & $\mathrm{Rb}-1$ & 90 & $47.26 \pm 5.44$ & Round \\
\hline 2 & $\mathrm{Rb}-2$ & 81.33 & $51.56 \pm 3.68$ & Oblong \\
\hline 3 & $\mathrm{Rb}-3$ & 82.33 & $108.16 \pm 6.42$ & Round \\
\hline 4 & $\mathrm{Rb}-4$ & 80 & $86.49 \pm 6.88$ & Round \\
\hline 5 & $\mathrm{Rb}-5$ & 75 & $93.03 \pm 15.37$ & Round \\
\hline 6 & $\mathrm{Rb}-6$ & 85.66 & $49.69 \pm 4.83$ & Round \\
\hline 7 & $\mathrm{Rb}-7$ & 78 & $50.06 \pm 4.95$ & Oblong \\
\hline 8 & $\mathrm{Rb}-8$ & 84.33 & $53.42 \pm 4.48$ & Round \\
\hline 9 & $\mathrm{Rb}-9$ & 83.66 & $63.14 \pm 7.64$ & Round \\
\hline 10 & $\mathrm{Rb}-10$ & 82 & $65.19 \pm 8.14$ & Round \\
\hline 11 & $\mathrm{Rb}-11$ & 90 & $46.14 \pm 6.91$ & Round \\
\hline 12 & $\mathrm{Rb}-12$ & 85.66 & $57.72 \pm 9.13$ & Round \\
\hline 13 & $\mathrm{Rb}-13$ & 76.33 & $58.66 \pm 9.34$ & Round \\
\hline 14 & $\mathrm{Rb}-14$ & 80 & $61.08 \pm 10.94$ & Round \\
\hline 15 & $\mathrm{Rb}-15$ & 90 & $68.74 \pm 5.64$ & Oblong \\
\hline 16 & $\mathrm{Rb}-16$ & 82.66 & $60.52 \pm 3.92$ & Round \\
\hline 17 & $\mathrm{Rb}-17$ & 80 & $61.64 \pm 10.78$ & Oblong \\
\hline 18 & $\mathrm{Rb}-18$ & 86 & $118.24 \pm 7.34$ & Round \\
\hline 19 & $\mathrm{Rb}-19$ & 83.33 & $46.33 \pm 6.59$ & Round \\
\hline 20 & $\mathrm{Rb}-20$ & 82 & $103.11 \pm 9.61$ & Round \\
\hline 21 & $\mathrm{Rb}-21$ & 76.33 & $44.27 \pm 5.57$ & Oblong \\
\hline 22 & $\mathrm{Rb}-22$ & 85.66 & $112.27 \pm 9.48$ & Round \\
\hline 23 & $\mathrm{Rb}-23$ & 90 & $61.27 \pm 9.07$ & Round \\
\hline 24 & $\mathrm{Rb}-24$ & 84.33 & $63.89 \pm 6.42$ & Round \\
\hline 25 & $\mathrm{Rb}-25$ & 78 & $56.23 \pm 6.80$ & Round \\
\hline 26 & $\mathrm{Rb}-26$ & 75 & $61.46 \pm 5.33$ & Round \\
\hline 27 & $\mathrm{Rb}-27$ & 82.33 & $117.87 \pm 13.08$ & Oblong \\
\hline 28 & $\mathrm{Rb}-28$ & 81.33 & $100.69 \pm 9.82$ & Round \\
\hline 29 & $\mathrm{Rb}-29$ & 90 & $93.03 \pm 15.37$ & Round \\
\hline 30 & $\mathrm{Rb}-30$ & 82 & $84.99 \pm 6.02$ & Round \\
\hline 31 & $\mathrm{Rb}-31$ & 79.33 & $86.49 \pm 6.88$ & Round \\
\hline 32 & $\mathrm{Rb}-32$ & 84.33 & $47.82 \pm 5.91$ & Round \\
\hline 33 & $\mathrm{Rb}-33$ & 82 & $120.11 \pm 7.68$ & Round \\
\hline 34 & $\mathrm{Rb}-34$ & 78.33 & $65.57 \pm 6.24$ & Round \\
\hline 35 & $\mathrm{Rb}-35$ & 85 & $54.92 \pm 6.21$ & Round \\
\hline 36 & $\mathrm{Rb}-36$ & 80 & $50.44 \pm 6.46$ & Round \\
\hline 37 & $\mathrm{Rb}-37$ & 85.66 & $116.19 \pm 13.36$ & Oblong \\
\hline 38 & $\mathrm{Rb}-38$ & 90 & $55.48 \pm 5.77$ & Round \\
\hline 39 & $\mathrm{Rb}-39$ & 82 & $80.88 \pm 4.61$ & Round \\
\hline 40 & $\mathrm{Rb}-40$ & 81.33 & $42.03 \pm 4.86$ & Oblong \\
\hline
\end{tabular}


Table.3 Categorization of $R$. bataticola isolates on the basis of radial growth

\begin{tabular}{|c|c|c|c|}
\hline Sr. No & Category & Number & Isolates \\
\hline 1 & Fast growing $(>80 \mathrm{~mm})$ & 32 & $\begin{array}{l}\text { Rb-1,Rb-2,Rb-3,Rb-4,Rb-6,Rb-8,Rb-9,Rb-10,Rb-11,Rb-12,Rb- } \\
\text { 14,Rb-15,Rb-16,Rb-17,Rb-18,Rb-19, Rb-20, Rb-22, Rb-23, Rb- } \\
\text { 24, Rb-27, Rb-28, Rb-29, Rb-30, Rb-32, Rb-33, Rb-35, Rb-36, } \\
\text { Rb-37,Rb-38, Rb-39, Rb-40 }\end{array}$ \\
\hline 2 & Medium growing $(70-80 \mathrm{~mm})$ & 8 & Rb-5, Rb-7, Rb-13, Rb-21, Rb-25, Rb-26, Rb-31, Rb-34 \\
\hline
\end{tabular}

Table.4 Categorization of $R$. bataticola isolates on the basis of size of sclerotia

\begin{tabular}{|c|c|c|l|}
\hline Sr. No & Category & Number & \multicolumn{1}{|c|}{ Isolates } \\
\hline $\mathbf{1}$ & Large sized $(>80 \mu \mathrm{m})$ & 16 & $\begin{array}{l}\text { Rb-3,Rb-4, Rb-5, Rb-18, Rb-20, Rb-22, Rb-23, Rb-24, Rb-27, Rb- } \\
28, \mathrm{Rb}-29, \mathrm{Rb}-30, \mathrm{Rb}-31, \mathrm{Rb}-33, \mathrm{Rb}-37, \mathrm{Rb}-39\end{array}$ \\
\hline $\mathbf{2}$ & Medium sized $(60-80 \mu \mathrm{m})$ & 8 & $\mathrm{Rb}-9, \mathrm{Rb}-10, \mathrm{Rb}-14, \mathrm{Rb}-15, \mathrm{Rb}-16, \mathrm{Rb}-17, \mathrm{Rb}-26, \mathrm{Rb}-34$ \\
\hline $\mathbf{3}$ & Small sized $(<60 \mu \mathrm{m})$ & 16 & $\begin{array}{l}\text { Rb-1, Rb-2, Rb-6, Rb-7, Rb-8, Rb-11, Rb-12, Rb-13, Rb-19, Rb- } \\
21, \mathrm{Rb}-25, \mathrm{Rb}-32, \mathrm{Rb}-35, \mathrm{Rb}-36, \mathrm{Rb}-38 \text { and Rb-40 }\end{array}$ \\
\hline
\end{tabular}

Table.5 Pathogenic variability among Rhizoctonia bataticola isolates against soybean cultivaTAMS-38

\begin{tabular}{|l|l|l|l|l|l|l|}
\hline Sr. No & Isolates & $\begin{array}{c}\text { Total no. } \\
\text { of seeds } \\
\text { Sown }\end{array}$ & $\begin{array}{c}\text { Total no. of } \\
\text { plants } \\
\text { germinated }\end{array}$ & $\begin{array}{c}\text { No. of } \\
\text { plants } \\
\text { infected }\end{array}$ & $\begin{array}{c}\text { Days to } \\
\text { initiate } \\
\text { symptoms }\end{array}$ & $\begin{array}{c}\text { Per cent } \\
\text { mortality duc to } \\
\text { pathogen (30 }\end{array}$ \\
\hline DAS)
\end{tabular}




\begin{tabular}{|c|c|c|c|c|c|c|}
\hline 23. & $\mathrm{Rb}-23$ & 60 & 48 & 22 & 18 & $45.83(42.60)$ \\
\hline 24. & $\mathrm{Rb}-24$ & 60 & 48 & 18 & 20 & $37.50(38.17)$ \\
\hline 25. & $\mathrm{Rb}-25$ & 60 & 47 & 23 & 18 & $48.94(44.37)$ \\
\hline 26. & $\mathrm{Rb}-26$ & 60 & 54 & 26 & 18 & $48.15(44.05)$ \\
\hline 27. & $\mathrm{Rb}-27$ & 60 & 48 & 23 & 18 & $47.92(43.80)$ \\
\hline 28. & $\mathrm{Rb}-28$ & 60 & 44 & 19 & 18 & $43.18(41.21)$ \\
\hline 29. & $\mathrm{Rb}-29$ & 60 & 45 & 23 & 17 & $51.11(46.04)$ \\
\hline 30. & $\mathrm{Rb}-30$ & 60 & 46 & 18 & 20 & $39.13(38.62)$ \\
\hline 31. & $\mathrm{Rb}-31$ & 60 & 51 & 19 & 20 & $37.25(37.40)$ \\
\hline 32. & $\mathrm{Rb}-32$ & 60 & 49 & 9 & 26 & $18.37(25.50)$ \\
\hline 33. & $\mathrm{Rb}-33$ & 60 & 49 & 21 & 18 & $42.86(41.03)$ \\
\hline 34. & $\mathrm{Rb}-34$ & 60 & 48 & 28 & 17 & $58.33(49.71)$ \\
\hline 35. & $\mathrm{Rb}-35$ & 60 & 51 & 26 & 17 & $50.98(45.63)$ \\
\hline 36. & $\mathrm{Rb}-36$ & 60 & 49 & 12 & 22 & $24.49(29.74)$ \\
\hline 37. & $\mathrm{Rb}-37$ & 60 & 44 & 17 & 20 & $38.64(38.45)$ \\
\hline 38. & $\mathrm{Rb}-38$ & 60 & 47 & 18 & 20 & $38.30(38.37)$ \\
\hline 39. & $\mathrm{Rb}-39$ & 60 & 51 & 30 & 17 & $58.82(50.03)$ \\
\hline 40. & $\mathrm{Rb}-40$ & 60 & 49 & 34 & 16 & $69.39(56.24)$ \\
\hline 41. & Control & 60 & 56 & 0 & 0 & $0(0)$ \\
\hline \multicolumn{6}{|c|}{ F Test } & Sig \\
\hline \multicolumn{6}{|c|}{ S.E (M) \pm} & 1.71 \\
\hline \multicolumn{6}{|c|}{ C.D $(p=0.05)$} & 4.81 \\
\hline
\end{tabular}

*Figures in parenthesis are arc sin value transformed

Table.6 Grouping of Rhizoctonia bataticola isolates based on their pathogenic ability

\begin{tabular}{|c|c|c|c|c|}
\hline $\begin{array}{l}\text { Sr. } \\
\text { No }\end{array}$ & Category & $\begin{array}{l}\text { Per cent }(\%) \\
\text { mortality due to } \\
\text { Root Rot }\end{array}$ & $\begin{array}{l}\text { No. of } \\
\text { Isolates }\end{array}$ & Isolates \\
\hline 1. & $\begin{array}{l}\text { Non-pathogenic } \\
\text { (NPI) }\end{array}$ & 0 & Nil & Nil \\
\hline 2 & $\begin{array}{l}\text { Weakly pathogenic } \\
\text { (WPI) }\end{array}$ & $1-20$ & 6 & $\begin{array}{l}\mathrm{Rb}-1, \mathrm{Rb}-2, \mathrm{Rb}-6, \mathrm{Rb}-7, \mathrm{Rb}- \\
19, \mathrm{Rb}-32\end{array}$ \\
\hline 3 & $\begin{array}{l}\text { Moderately pathogenic } \\
\text { (MPI) }\end{array}$ & $21-50$ & 24 & $\begin{array}{l}\mathrm{Rb}-4, \mathrm{Rb}-5, \mathrm{Rb}-8, \mathrm{Rb}-9, \mathrm{Rb}- \\
11, \mathrm{Rb}-12, \mathrm{Rb}-14, \mathrm{Rb}-15, \mathrm{Rb}- \\
16, \mathrm{Rb}-20, \mathrm{Rb}-21, \mathrm{Rb}-23, \mathrm{Rb}- \\
24, \mathrm{Rb}-25, \mathrm{Rb}-26, \mathrm{Rb}-27, \mathrm{Rb}- \\
28, \mathrm{Rb}-30, \mathrm{Rb}-31, \mathrm{Rb}-33, \mathrm{Rb}- \\
35, \mathrm{Rb}-36, \mathrm{Rb}-37, \mathrm{Rb}-38\end{array}$ \\
\hline 4 & $\begin{array}{l}\text { Strongly pathogenic } \\
\text { (SPI) }\end{array}$ & $51-70$ & 8 & $\begin{array}{l}\mathrm{Rb}-10, \mathrm{Rb}-13, \mathrm{Rb}-18, \mathrm{Rb}-22 \\
\mathrm{Rb}-29, \mathrm{Rb}-34, \mathrm{Rb}-39, \mathrm{Rb}-40\end{array}$ \\
\hline 5 & $\begin{array}{l}\text { Highly pathogenic } \\
\text { (HPI) }\end{array}$ & $>70$ & 2 & $\mathrm{Rb}-3, \mathrm{Rb}-17$ \\
\hline
\end{tabular}


Table.7 Reaction of host differentials to Rhizoctonia bataticola under sick soil condition

\begin{tabular}{|c|c|c|c|c|c|c|c|}
\hline \multirow[t]{2}{*}{ Cultivar } & \multicolumn{7}{|c|}{ Rhizoctonia bataticola isolates } \\
\hline & Rb-39 & Rb-38 & Rb-29 & $\mathbf{R b}-22$ & $\mathbf{R b}-\mathbf{1 0}$ & $\mathbf{R b}-17$ & $\mathbf{R b}-\mathbf{3}$ \\
\hline \multirow[t]{2}{*}{ GBIC-18758 } & $85.71 *$ & 82.35 & 64.29 & 70.00 & 35.29 & 9.52 & 46.15 \\
\hline & HS & HS & $\mathrm{S}$ & $\mathrm{S}$ & MS & HR & MS \\
\hline \multirow[t]{2}{*}{ AMS-595 } & 75.00 & 76.47 & 100.00 & 66.67 & 80.00 & 46.15 & 83.33 \\
\hline & $\mathrm{S}$ & $\mathrm{S}$ & HS & $\mathrm{S}$ & HS & MS & HS \\
\hline \multirow[t]{2}{*}{ AMS-MB-5-18 } & 100.00 & 53.85 & 50.00 & 31.25 & 100.00 & 21.74 & 75.00 \\
\hline & HS & MS & MS & MR & HS & MR & S \\
\hline \multirow[t]{2}{*}{ AMS-MB-5-19 } & 16.67 & 50.00 & 46.15 & 22.22 & 50.00 & 45.00 & 47.37 \\
\hline & MR & MS & MS & MR & MS & MS & MS \\
\hline \multirow[t]{2}{*}{ AMS-1003 } & 81.82 & 18.18 & 88.89 & 75.00 & 21.43 & 42.86 & 66.67 \\
\hline & HS & MR & HS & $\mathrm{S}$ & MR & MS & MS \\
\hline \multirow[t]{2}{*}{ AMS-475 } & 72.22 & 57.14 & 100.00 & 75.00 & 66.67 & 66.67 & 100.00 \\
\hline & $\mathrm{S}$ & $\mathrm{S}$ & HS & $\mathrm{S}$ & $\mathrm{S}$ & $\mathrm{S}$ & HS \\
\hline \multirow[t]{2}{*}{ AMS-3923 } & 60.71 & 57.14 & 85.71 & 21.43 & 57.14 & 43.75 & 64.00 \\
\hline & $\mathrm{S}$ & $\mathrm{S}$ & HS & MR & S & MS & S \\
\hline \multirow[t]{2}{*}{ AMS-9933 } & 22.73 & 60.00 & 90.91 & 71.43 & 75.00 & 65.00 & 42.11 \\
\hline & MR & $\mathrm{S}$ & HS & $\mathrm{S}$ & $\mathrm{S}$ & $\mathrm{S}$ & MS \\
\hline \multirow[t]{2}{*}{ Bragg } & 61.54 & 61.11 & 78.57 & 42.86 & 69.23 & 64.71 & 50.00 \\
\hline & S & S & HS & MS & S & S & MS \\
\hline \multirow[t]{2}{*}{ Punjab-1 } & 68.75 & 46.67 & 80.00 & 40.00 & 41.67 & 42.86 & 63.64 \\
\hline & S & MS & HS & MS & MS & MS & S \\
\hline \multirow[t]{2}{*}{ TAMS-38 } & 71.43 & 53.85 & 63.64 & 81.82 & 50.00 & 75.00 & 78.57 \\
\hline & $\mathrm{S}$ & MS & $\mathrm{S}$ & HS & MS & $\mathrm{S}$ & HS \\
\hline \multirow[t]{2}{*}{ TAMS-98-21 } & 71.43 & 66.67 & 100.00 & 83.33 & 81.82 & 76.47 & 76.47 \\
\hline & $\mathrm{S}$ & $\mathrm{S}$ & HS & HS & HS & S & S \\
\hline
\end{tabular}

* Per cent disease incidence

MR- Moderately Resistant (12.22-33.33 \% mortality), MS- Moderately Susceptible (34.44-55.55 \% mortality), S- Susceptible (56.66-77.77 \% mortality), HSHighly Susceptible (78.88-100.00 \% mortality) 
AMS-475 genotype was highly susceptible to isolates $\mathrm{Rb}-29$ (100.00 \%) and Rb-3 (100.00 $\%)$ indicating highest virulence followed by isolates Rb-39 (72.22 \%), Rb-22 (75.00\%), Rb-38 (57.14 \%), Rb-10 (66.67 \%) and Rb-17 (66.67 \%). AMS-3923 showed moderate resistance against $\mathrm{Rb}-22$ (21.43 \%) while moderately susceptible reaction against $\mathrm{Rb}-17$ (43.75 \%). Isolates Rb-38 (60.00\%), Rb-22 (71.43 \%), Rb-10 (75.00 \%) and Rb-17 (65.00 $\%)$ exhibited more virulence against AMS9933. At the same time, same genotype was moderately resistant to isolate $\mathrm{Rb}-39$ (22.73 $\%$ ). Soybean Bragg showed susceptible to highly susceptible reaction against all isolates. Three isolates viz., Rb-38 (46.67\%), Rb-22 (40.00 \%), Rb-10 (41.67 \%) and Rb-17 (42.86 $\%)$ exhibited moderately susceptible reaction against Panjab-1. Soybean TAMS-38 was highly susceptible to isolate Rb-22 (81.82\%) and Rb-3 (78.57 \%) indicating highest virulence followed by isolate $\mathrm{Rb}-39$ (71.43 $\%), \mathrm{Rb}-29$ (63.64 \%) and Rb-17 (75.00\%) which showed susceptible reaction. TAMS98-21 showed highest susceptible reaction against Rb-29 (100.00 \%), Rb-22 (83.33 \%) and $\mathrm{Rb}-10$ (81.82 \%) followed by Rb-39 (71.43\%), Rb-38 (66.67\%), Rb-17 (76.47\%) and Rb-3 (76.47 \%) which showed susceptible reaction.

$\mathrm{Rb}$-29 isolate showed broad virulence range as it infected all the genotypes followed by isolate $\mathrm{Rb}-38$ which infected eleven genotypes. AMS-MB-5-18 and AMS-MB-519 were moderately resistance to three isolates $\mathrm{Rb}-22, \mathrm{Rb}-17$ and $\mathrm{Rb}-39$ which were not able to cause severe infection. In present study, isolate from Kurundwad, Sangli ( $\mathrm{Rb}$ 29) has exhibited constant severe pathogenic reaction in most of the tested entries. So this isolate will be useful for evaluation of resistance in soybean germplasm and breeding lines and in resistance breeding programmed against root rot of soybean. Seven isolates of $R$. bataticola isolated from diversed locations exhibit the different pathogenic reaction against tested. entries. It indicated considerable variability in tested isolates. This showed physiological specialization within $R$. bataticola.

In the present studies, 40 isolates of $R$. bataticola belongs to different soybean growing states of India showed variations in different morphological traits viz., radial growth, sclerotial size as well as in pathogenicity. The variations in morphology might be due to differences in temperature, moisture, soil types, and other edaphic factors of various places (Iqbal and Mukhtar, 2014). Morphological variability has also been reported by many workers in terms of growth, color, pycnidium production, and chlorate sensitivity among different isolates of $M$. phaseolina on different hosts (Dhingra and Sinclair, 1973; Pearson et al., 1986; Atiq et al., 2001 and Riaz et al., 2007) which corroborated our findings. However, in the present studies, no relationship was found among the morphological characters and pathogenicity of the isolates.

Thirty two isolates showed radial growths above $80 \mathrm{~mm}$ and were rated as fast growing while rest of the isolates showed growth between 70 and $80 \mathrm{~mm}$ and hence were classified as medium growing. The size of sclerotia of 16 isolates was above $80 \mu \mathrm{m}$ and was classified as large sized while 16 isolates with sclerotial size less than $60 \mu \mathrm{m}$ were rated as small sized. The remaining 8 isolates ranged between 60 and $80 \mu \mathrm{m}$ sclerotial size and were categorized as medium sized. Earlier workers while working on $R$. bataticola associated with different crop plants also grouped the isolates into different categories based on colony characters on medium (Raut and Ingle, 1989; Meena Shekhar et al., 2006; Aghakhani and Dubey, 2009; Sharma et al., 2012 and Datta et al., 2013). 
The pathogenic fungus, M. phaseolina, has a broad host range and exits in two asexual forms which maintain its survival better (Dhingra and Sinclair, 1978; Cloud and Rupe, 1988). Some workers also related variability to the phenomena of host specialization in $M$. phaseolina. Su et al., (2001) found host specialization in maize on the basis of pathogenic, genetic, and physiological differences. Similarly, Cloud and Rupe (1988) analyzed host specialization in soybean.

This mechanism takes long time to establish within a specific host. Mihail and Taylor (1995) suggested that, due to heterogenic nature of M. phaseolina, categorization into distinct subgroups based upon pathogenicity and morphology could not take place. Pathogenesis along with genetic diversity plays a specific role in host-plant resistance. Isolates having morphological similarity are not necessarily identical genetically, they might have some differences. The variable genetic pattern contributes to variation in morphology and pathogenesis, which has been confirmed by using different molecular tools (Mayek-P'erez et al., 2001; ReyesFranco et al., 2006; Almeida et al., 2003 and Jana et al., 2003). As the pathogen has no sexual phase, genetic diversity is produced either by fusion of vegetative cells or by parasexual recombination between nuclear genes (Carlile, 1986). In nature genetic variability improves survival of a fungus (Bashasab, and Kuruvinashetti, 2007).

It is quite evident that variability in morphology, physiology, genetics, pathogenicity, and so forth is imperative for the fungus to have better adaptation in response to diversified environmental behavior. It also leads to host-plant resistance, development of resistant varieties of different crops against disease, and implementation of new disease controlling strategies (Purkayastha et al., 2006).
The determination of variability among $R$. bataticola isolates is fundamental to guide the development of appropriate strategies for disease management according to different agro ecological zones. The present studies provide information on the variability of $R$. bataticola in major soybean growing states of India. These results will be useful in developing integrated strategies for the management of soybean root rot and breeding programs for pulses and other crops.

\section{References}

Abawi, G.S. and M. A. Pastor-Corrales. 1990. Root Rots of Beans in Latin America and Africa: Diagnosis, Research Methodologies and Management Strategies, CIAT, Cali, Colombia.

Aghakhani Maryam and S.C. Dubey. 2009. Morphological and pathogenic variation among isolates of Rhizoctonia bataticola causing dry root rot of chickpea. Indian Phytopath. 62 (2): 183189.

Almeida, A. M. R., R. V. Abdelnoor, C. A. A. Arias. 2003. Genotypic diversity among Brazilian isolates of Macrophomina phaseolina revealed by RAPD. Fitopatologia Brasileira. 28 (3): 279285.

Atiq, M., A. Shabeer and I. Ahmed. 2001. Pathogenic and cultural variation in Macrophomina phaseolina, the cause of charcoal rot in sunflower. Sarhad Journal of Agriculture. 2: 253-255.

Carlile, M. J. 1986. Genetic exchange and gene flow: their promotion and prevention. p. 203-214. In: A. D. M. Rayner, C. M. Brasier, and D. Moore, (ed). Evolutionary Biological of the Fungi. Cambridge University Press, Cambridge, UK.

Cloud G. L. and J. C. Rupe. 1988. Preferential host selection of isolates of Macrophomina phaseolina. 
Phytopathology. 78 (12): 1563.

Datta Upma, Sachin Gupta, C. S. Kalha and V. K. Razdan. 2013. Morpho cultural and pathogenic variability isolates Rhizoctonia solani causing sheath blight of rice in Jammu. J. Mycol. Pl. Pathol.43 (2): 210-215.

Dhingra, O. D. and D. Chagas. 1981. Effect of soil temperature, moisture and nitrogen on competitive saprophytic ability of Macrophomina phaseolina. Transactions of British Mycological Society. 77 (1): 15-20.

Dhingra, O. D. and J. B. Sinclair. 1973. Location of Macrophomina phaseolina on soybean plants related to cultural characteristics and virulence. Phytopathology. 63 (7): 934-936.

Dhingra, O. D. and J. B. Sinclair. 1978. Biology and Pathology of Macrophomina phaseolina. Universidade Federal de Vicosa, Minas Gerais, Brazil.

Jana, T., T. R. Sharma, R.D. Prasad and D. K. Arora. 2003. Molecular characterization of Macrophomina phaseolina and Fusarium species by a single primer RAPD technique. Microbiological Research. 158 (3): 249-257.

Mayek-P'erez, N., C. L'opez-Casta neda, M. Gonz'alez-Chavira. 2001. Variability of Mexican isolates of Macrophomina phaseolina on basis of pathogenesis and AFLP genotype. Physiological and Molecular Plant Pathology. 59 (5): 257 264.

Meena Shekhar, R. C. Sharma, Sujay Rakshit, Poonam Yadav, Lokendra Singh and Ram Dutta, 2006. Genetic variability in Macrophomina phaseolina (Tassi.) Goid. incitant of charcoal rot of maize in India. Indian Phytopath. 59 (4): 453 459.

Mihail, J. D. and S. J. Taylor. 1995. Interpreting variability among isolates of Macrophomina phaseolina in pathogenicity, pycnidium production, and chlorate utilization. Canadian Journal of Botany.73 (10): 1596-1603.

Papavizas, G. C. 1977. Some factors affecting survival of sclerotia of Macrophomina phaseolina in soil. Soil Biology and Biochemistry. 9 (5): 337-341.

Pawar, N. B. 2010. Pathogenic variability among the isolates of chickpea wilt complex. Ph. D diss., Dept. Plant Path., Dr. Panjabrao Deshmukh Krishi Vidyapeeth, Akola, Maharashtra, India.

Pearson, C. A. S., J. F. Leslie and F. W. Schwenk. 1986. Variable chlorate resistance in Macrophomina phaseolina from corn, soybean, and soil. Phytopathology. 76 (6) 646-649.

Purkayastha, S., B. Kaur, N. Dilbaghi and A. Chaudhury. 2006. Characterization of Macrophomina phaseolina, the charcoal rot pathogen of cluster bean, using conventional techniques and PCR-based molecular markers. Plant Pathol. 55: 106- 116.

Rajkumar, F. Bashasab, and M. S. Kuruvinashetti, 2007. Genetic variability of sorghum charcoal rot pathogen (Macrophomina phaseolina) assessed by random DNA markers. Plant Pathology. 23: 45-50.

Raut, J.G. and R.W. Ingle. 1989. Variations in isolates of Rhizoctonia bataticola. Indian Phytopath. 42 (4): 506-508.

Reyes-Franco, M. C., S. Hern'andez-Delgado, R. Beas- Fern'andez, M. MedinaFern'andez, J. Simpson, and N. MayekP'erez. 2006. Pathogenic and genetic variability within Macrophomina phaseolina from Mexico and other countries. Journal of Phytopathology. 154(7-8): 447-453.

Riaz A., S. H. Khan, S.M.Iqbal and M. Shoaib. 2007. Pathogenic variability among Macrophomina phaseolina (Tassi) Goid, isolates and identification of sources of resistance in mash against 
charcoal rot. Pakistan Journal of Phytopathology. 19 (1): 44-46.

Sharma Mamta, Raju Ghosh, Ramesh R. Krishnan, Upala N. Nagamangala, Shiva Chamarthi, Rajeev Varshney and Suresh Pande, 2012. Molecular and morphological diversity in Rhizoctonia bataticola isolates causing dry root rot of chickpea (Cicer arietinum L.) in India. African Journal of Biotechnology. 11 (37): 8948-8959.

Sinclair J. B. 1982. Compendium of Soybean Diseases. 2nd ed. American Phytopathological Society, Saint Paul, Minn, USA.

Sinclair, J. B. and M. C. Shurlleff. 1975. Compendium of soybean diseases. Amer. Phytopath. Soc. Inc. Minnesota, USA: 8-10.

Su, G., S. O. Suh, R.W. Schneider and J. S. Russin. 2001. Host specialization in the charcoal rot fungus, Macrophomina phaseolina. Phytopathology. 91 (2): 120-126.

Umer Iqbal and Tariq Mukhtar. 2014. Morphological and pathogenic variability among Macrophomina phaseolina isolates associated with Mungbean (Vigna radiata L.) Wilczek from Pakistan. Scientific World Journal. Article ID: 950175. pages 9.

Wheeler, H. 1975. Plant Pathogenesis, Academic press, London, UK.

Wrather, J. Allen and Steve R. Koenning, 2006. Estimates of disease effects on soybean yields in the United States 2003-2005. J. of Nematology. 38 (2):173-180.

Yang, X. B. and S. S. Navi. 2005. First report of charcoal rot epidemics caused by Macrophomina phaseolina in soybean, in IOWA. Pl. Dis. 89(5): 26.

\section{How to cite this article:}

Gade, R.M., Y.K. Belkar and Ingle, Y.V. 2018. Morphological And Pathogenic Variability among Rhizoctonia bataticola Isolates Associated with Soybean (Glycine max L.) from India. Int.J.Curr.Microbiol.App.Sci. 7(01): 2575-2588. doi: https://doi.org/10.20546/ijcmas.2018.701.310 